\title{
Electromotive force and internal resistance of an electron pump
}

\author{
Miguel Rey $*$ and Fernando Sol: \\ Departamento de Física Teórica de la Materia Condensada and Instituto Nicolás Cabrera, \\ Universidad Autónoma de Madrid, E-28049 Madrid, Spain
}

(Dated: November 20, 2018)

\begin{abstract}
We present a scattering theory of the electromotive force and internal resistance of an electron pump. The characterization of the device performance in terms of only two parameters requires the assumption of incoherent multiple scattering within the circuit and complete thermalization among electrons moving in a given direction. The electromotive force is shown to be of the order of the driving frequency in natural units. In an open setup, the electromotive force adds to the voltage difference between reservoirs to drive the current, both facing a contact resistance which is absent in the case of a closed circuit of uniform width.
\end{abstract}

Electrons pumps favor electron motion in a given direction by combining nonlinear ac driving and some asymmetry in the spatial structure or in the temporal signal. This rectification of electron motion generates current in the absence of a net dc voltage bias $1,2,23,4,5,6,6,6]$. Most theoretical calculations have dealt so far with the calculation of the pump current, which is the current flowing through the device when the electrons incident from both sides are characterized by the same chemical potential. In practice, however, one is likely to be interested in the performance of the pump as a circuit component, something which cannot be predicted from the mere knowledge of the pump current. This creates the need to characterize the electron pump as a battery with a certain electromotive force and internal resistance. Although the electromotive force can in principle be obtained from the dc bias that exactly cancels the pump current [1, 6], its derivation within a unified and general scheme seems desirable. On the other hand, there is no obvious ansatz for the calculation of the internal resistance. A potential application of this new class of devices is the generation of current in small closed circuits not attached to broad wires acting as electron reservoirs. Such a setup is schematically depicted in Fig. 1. One may also consider a pump in series with a resistor, both within a lead that couples to large reservoirs through ideal contacts, as indicated in Fig. 2. In this article, we derive formulae which express the battery parameters in terms of the transmission and reflection probabilities for electrons crossing the pump and the resistor. The scattering theory here presented attempts to play a role for the electromotive force and internal resistance of an electron pump similar to that which the LandauerBüttiker theory has represented for the conductance of nanostructures [9]. An important difference, however, is that the scenario which we investigate requires a more coarse-grained description if we wish to uniquely characterize the circuit performance of the pump in terms of a small set of parameters. Such an effective self-averaging of the device performance requires the assumption of electron decoherence between the pump and the resistor in series. Unlike in Ref. [10], we assume that effective phase randomization can be achieved with negligible backscattering. This hypothesis is supported by the fact that a minor distortion of the environment suffices to induce electron dephasing, while a more continued interaction is needed to change the electron energy or direction appreciably [1]. We find that reflectionless decoherence between the circuit elements is still insufficient to permit the characterization in terms of only two parameters, and not twice as many as available transverse channels. To achieve a simple and manageable description we must assume that, within the leads, electrons moving in each direction are characterized by a single chemical potential. The adequacy of this assumption, or its replacement by a weaker one within a model of comparable tractability, deserves further study. The total current through a two-lead multimode structure in the presence of local ac driving may be written

$$
\begin{gathered}
I=\frac{e}{h} \int d E_{i}\left[f\left(E_{i}-\mu_{R}^{\mathrm{in}}\right) T_{L R}\left(E_{i}\right)-f\left(E_{i}-\mu_{L}^{\mathrm{in}}\right) T_{R L}\left(E_{i}\right)\right] \\
T_{L R}\left(E_{i}\right) \equiv \sum_{a \in L} \sum_{b \in R} \int d E_{f} T_{a b}^{L R}\left(E_{f}, E_{i}\right),
\end{gathered}
$$

$T_{a b}^{L R}\left(E_{f}, E_{i}\right)$ being the probability distribution that an electron incident from the right lead in channel $b$ with energy $E_{i}$ is transmitted into channel $a$ of the left lead with energy $E_{f}$. For future convenience, we assume $I>0$ when current flows from right to left. The chemical potentials $\mu_{L}^{\text {in }}, \mu_{R}^{\text {in }}$ characterize the population of incoming electrons. The pump effect is based on the existence of an asymmetry between the left-to-right and right-to-left transmissions. Thus it is convenient to define:

$$
\begin{aligned}
T\left(E_{i}\right) & \equiv\left[T_{L R}\left(E_{i}\right)+T_{R L}\left(E_{i}\right)\right] / 2 \\
\delta T\left(E_{i}\right) & \equiv T_{L R}\left(E_{i}\right)-T_{R L}\left(E_{i}\right) .
\end{aligned}
$$

If we linearize $f\left(E-\mu_{L, R}^{\mathrm{in}}\right)$ around a common reference chemical potential $\mu_{0}$, we may write the total current as the sum of a bias and a pump contribution

$$
I=I_{B}+I_{P}
$$




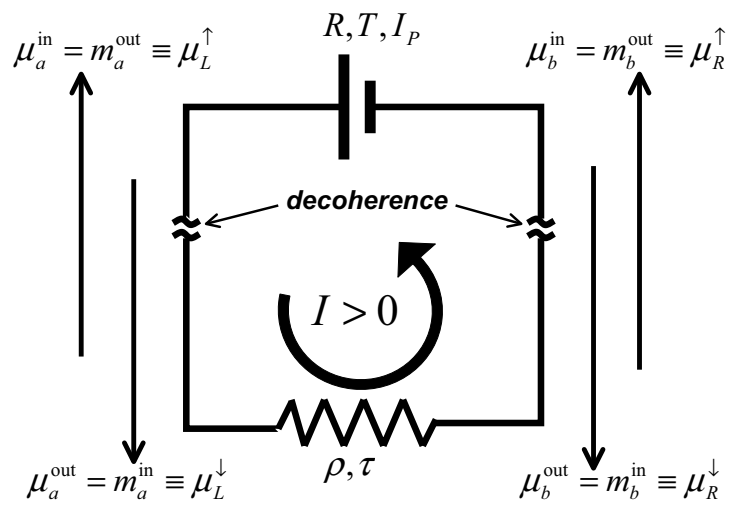

FIG. 1: Schematic representation of an electron pump in series with a generic resistor within a closed circuit where current flows thanks to the action of the pump

$$
\begin{aligned}
I_{B} & \equiv \frac{e}{h} \Delta \mu^{\text {in }} \int d E_{i}\left[-f^{\prime}\left(E_{i}-\mu_{0}\right)\right] T\left(E_{i}\right) \\
I_{P} & \equiv \frac{e}{h} \int f\left(E_{i}-\mu_{0}\right) \delta T\left(E_{i}\right)
\end{aligned}
$$

with $\Delta \mu^{\text {in }} \equiv \mu_{R}^{\text {in }}-\mu_{L}^{\text {in }}$. Hereafter, we take $\mu_{0} \equiv 0$, although we note that (unlike $\left.I_{B}\right) I_{P}$ does depend on $\mu_{0}$. Let us focus on the current flow in a given channel $a$ on e.g. lead $L$. For convenience, we define $\tilde{I}_{\alpha} \equiv(h / e) I_{\alpha}$ for all future current contributions. To achieve a better perspective, we momentarily abandon the assumption that the chemical potential is channel independent. The total current through channel $a \in L$ can then be written:

$$
\tilde{I}_{a}=-\mu_{a}^{\text {in }}+\mu_{a}^{\text {out }},
$$

where $\mu_{a}^{\text {in(out) }}$ characterizes the population of electrons in $L$ approaching (leaving) the pump. We note that, even if the "in" population is rigorously thermal, the "out" population is not. However, one can always find a suitably defined chemical chemical potential $\mu_{a}^{\text {out }}$ that reproduces the same current flow (and, in one dimension, the same current density [12]). Like the total current, this outgoing chemical potential has a "bias" and a "pump" contribution,

$$
\begin{aligned}
\mu_{a}^{\text {out }} & =\mu_{a}^{\text {out }, \mathrm{B}}+\mu_{a}^{\text {out }, \mathrm{P}} \\
\mu_{a}^{\text {out }, \mathrm{B}} & =\sum_{b} S_{a b} \mu_{b}^{\text {in }} \\
\mu_{a}^{\text {out }, \mathrm{P}} & =\tilde{I}_{P, a},
\end{aligned}
$$

where $\tilde{I}_{P, a}$ is the pump current in channel $a\left(\sum_{a} \tilde{I}_{P, a}=\right.$ $\left.\tilde{I}_{P}\right)$. Since the bias contribution depends only on the symmetrized probability [see Eq. [3)], we have $S_{a b}=S_{b a}$. On the other hand, unitarity requires $\sum_{b} S_{a b}=1$. The term $\mu_{a}^{\text {out,P }}$ accounts for the excess (or defect) of electrons generated by the pump. It reflects the fact that an

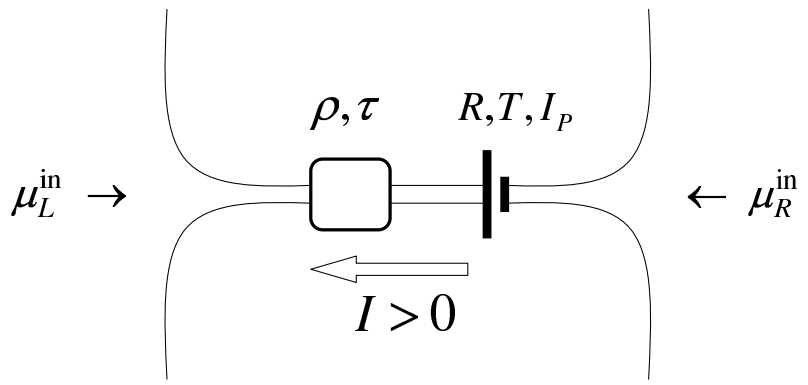

FIG. 2: Electron pump in series with a scattering barrier, both within a multichannel wire that couples through ideal contacts to large electron reservoirs. Both the action of the pump and the difference between the reservoir chemical potentials contribute to drive the current through the wire

operating battery creates a population imbalance which ultimately drives the current through the circuit. Assume that, in series with the pump, we introduce a resistor which is also characterized in terms of its scattering probabilities. The resulting circuit is schematically depicted in Fig. 1. Being the resistor a passive element, its flow equations do not include a pump term. We write

$$
\tilde{I}_{a}=m_{a}^{\text {in }}-\sum_{b} \sigma_{a b} m_{b}^{\text {in }} \equiv m_{a}^{\text {in }}-m_{a}^{\text {out }},
$$

where $m_{a}^{\text {in (out) }}$ is the chemical potential for the electrons approaching (leaving) the resistor, and $\left\{\sigma_{a b}\right\}$ are the scattering probabilities by the resistor, which obey $\sigma_{a b}=\sigma_{b a}$ and $\sum_{a} \sigma_{a b}=1$. The sign convention in Eq. (12) is different from that used in the pump equations because counterclockwise current is taken to be positive (see Fig. 1). Now we note that the "out" population of the pump is the "in" population of the resistor, and viceversa. We seal this equivalence by establishing a common notation. For $a \in L$ we write

$$
\begin{gathered}
\mu_{a}^{\text {in }}=m_{a}^{\text {out }} \equiv \mu_{L, a}^{\uparrow} \\
\mu_{a}^{\text {out }}=m_{a}^{\text {in }} \equiv \mu_{L, a}^{\downarrow},
\end{gathered}
$$

and similarly for $a \in R$. The vertical arrows refer to the direction of movement within the convention of Fig. 1. Although a solution of the flow equations that would permit us to predict the total current in terms of $\left\{I_{P, a}, S_{a b}, \sigma_{a b}\right\}$ is formally possible, our real goal is the characterization of the pump in terms of two parameters. To achieve this objective, we have to introduce the simplifying assumption that electrons flowing in a given direction within a lead are all characterized by the same chemical potential. We express it as

$$
\mu_{L, a}^{\uparrow \downarrow}=\mu_{L}^{\uparrow \downarrow} \quad \mu_{R, a}^{\uparrow \downarrow}=\mu_{R}^{\uparrow \downarrow} \quad \forall a \in L, R
$$

Hereafter we differentiate between reflection and transmission probabilities: $S_{a b} \rightarrow R_{a b}, T_{a b}$ and $\sigma_{a b} \rightarrow \rho_{a b}, \tau_{a b}$. 
We introduce the notation

$$
\begin{array}{r}
R_{a} \equiv \sum_{b} R_{a b} \quad T_{a} \equiv \sum_{b} T_{a b} \quad\left(R_{a}+T_{a}=1\right) \\
R \equiv \sum_{a} R_{a} \quad T \equiv \sum_{a} T_{a} \quad(R+T=N)
\end{array}
$$

$N$ being the number of transverse channels. The resistor parameters $\rho_{a}, \tau_{a}, \rho, \tau$ are defined analogously. We introduce an average "pump chemical potential" $\mu_{P} \equiv$ $\sum_{a} \mu_{a}^{\text {out }, \mathrm{P}} / N=\tilde{I}_{P} / N$. The total current can now be written

$$
\tilde{I}=N\left(\mu_{L}^{\downarrow}-\mu_{L}^{\uparrow}\right)=N\left(\mu_{R}^{\uparrow}-\mu_{R}^{\downarrow}\right)
$$

These four chemical potential are not independent but are rather related by the flow equations

$$
\begin{aligned}
& N \mu_{L}^{\downarrow}=R \mu_{L}^{\uparrow}+T \mu_{R}^{\uparrow}+N \mu_{P} \\
& N \mu_{L}^{\uparrow}=\rho \mu_{L}^{\downarrow}+\tau \mu_{R}^{\downarrow} \\
& N \mu_{R}^{\downarrow}=T \mu_{L}^{\uparrow}+R \mu_{R}^{\uparrow}-N \mu_{P} \\
& N \mu_{R}^{\uparrow}=\tau \mu_{L}^{\downarrow}+\rho \mu_{R}^{\downarrow},
\end{aligned}
$$

which are physically transparent. The different sign carried by the pump contribution $N \mu_{P}$ in Eqs. (19) and (21) expresses the fact that, when $\mu_{P}>0$, there is an excess of outgoing electrons on the left of the pump and a corresponding defect of outgoing electrons on the right. When Eqs. (19) - (22) are introduced into Eq. (18), we obtain for the total current

$$
\begin{aligned}
\tilde{I} & =-T \Delta \mu^{\uparrow}+N \mu_{P} \\
& =\tau \Delta \mu^{\downarrow},
\end{aligned}
$$

where the chemical potential differences $\Delta \mu^{\uparrow \downarrow} \equiv \mu_{L}^{\uparrow \downarrow}-\mu_{R}^{\uparrow \downarrow}$ satisfy the relations

$$
\begin{aligned}
& N \Delta \mu^{\downarrow}=(R-T) \Delta \mu^{\uparrow}+2 N \mu_{P} \\
& N \Delta \mu^{\uparrow}=(\rho-\tau) \Delta \mu^{\downarrow} . .
\end{aligned}
$$

We may solve for $\Delta \mu^{\uparrow \downarrow}$ in Eqs. (25) and (26) and introduce the solutions in either (23) or (24) to obtain

$$
\tilde{I}=\frac{(N / T) I_{P}}{\rho / \tau+R / T} .
$$

Calculating the electromotive force $\mathcal{V}_{\text {emf }}$ and the internal resistance $\mathcal{R}_{i}$ amounts to finding a relation

$$
I=\frac{\mathcal{V}_{\mathrm{emf}}}{\mathcal{R}+\mathcal{R}_{i}},
$$

where $\mathcal{R}$ is a suitably defined resistance for the resistor. Comparison of Eqs. (27) and (28) uniquely leads to the result

$$
\begin{aligned}
\mathcal{V}_{\mathrm{emf}} & =\frac{h}{e^{2}} \frac{I_{P}}{T} \\
\mathcal{R}_{i} & =\frac{h}{N e^{2}} \frac{R}{T}
\end{aligned}
$$

provided that

$$
\mathcal{R}=\frac{h}{N e^{2}} \frac{\rho}{\tau} .
$$

The prefactors have been chosen to make $\mathcal{V}_{\text {emf }}$ an intensive quantity while $\mathcal{R}, \mathcal{R}_{i} \sim N^{-1}$ as $N \rightarrow \infty$. We may apply our results for $\mathcal{V}_{\text {emf }}$ and $\mathcal{R}_{i}$ to the analytically solvable pipeline model, which assumes that transmission takes place only within a single pair of channels [6]. It can be expressed as:

$$
T_{a b}^{L R}\left(E_{f}, E_{i}\right)=J \delta_{a b} \delta\left(E_{f}^{z}-E_{2}\right) \delta\left(E_{i}^{z}-E_{1}\right) .
$$

Here $E_{\alpha}^{z}(\alpha=i, f)$ is the energy in the direction perpendicular to the planar structure and $\left(E_{2}-E_{1}\right) / \hbar=$ $\omega>0$ is the driving frequency. The other scattering probabilities are determined by time-reversal symmetry in the presence of coherent ac driving $\left[T_{a b}^{L R}\left(E, E^{\prime}\right)=\right.$ $\left.T_{b a}^{R L}\left(E^{\prime}, E\right)\right]$ and unitarity. For three dimensions, the single pipeline model yields

$$
T=D J
$$

where $D=A m / 2 \pi \hbar^{2}$ is the two-dimensional transverse density of states, $A$ being the interface area. Preservation of unitarity requires $D J<N$. The total pump current is

$$
I_{P}=e D J \omega / 2 \pi
$$

so we interpret $e D J$ as the pumped charge per cycle. For the circuit parameters we obtain

$$
\begin{aligned}
\mathcal{V}_{\mathrm{emf}} & =\hbar \omega / e \\
\mathcal{R}_{i} & =\frac{h}{N e^{2}} \frac{N-D J}{D J}
\end{aligned}
$$

The result that the electromotive force is just $\hbar \omega / e$, independently of the transmittivity $J$, is remarkable if one looks at the general structure of Eqs. (77), (16), (17), and (29), but could have been expected from the notion that the pipeline model allows only for an energy gain $\hbar \omega$ as the electron is pumped from right to left, regardless of the total electron flow. We readily conclude that, in a more general pump structure, $\mathcal{V}_{\mathrm{emf}} \sim \hbar \omega / e$, in agreement with Refs. [1, 6]. By contrast, the internal resistance is very sensitive to the transmittivity of the pump. In particular, we note that $\mathcal{R}_{i} \rightarrow \infty$ as $J \rightarrow 0$. We now turn our attention to an open setup where the pump and resistor stay in series within a lead coupled through ideal contacts to broad electron reservoirs. As indicated in Fig. 2 , the chemical potentials in the reservoirs characterize the population of the incoming electrons. Hence, we refer to them also as $\mu_{L, R}^{\text {in }}$. One may perform an analysis similar to that described for the closed geometry of Fig. 1. After some algebra, one obtains

$$
I=(e / h) T^{\prime}\left(e \mathcal{V}_{\mathrm{emf}}+\Delta \mu^{\mathrm{in}}\right),
$$


where $\mathcal{V}_{\text {emf }}$ is given by Eq. (29), and $T^{\prime} \equiv(T \tau / N) /(1-$ $\left.R \rho / N^{2}\right)$ is the the average transmission through the compound structure formed by the pump plus the resistor. Interestingly, Eq. (37) can also be written as

$$
I=\frac{\mathcal{V}_{\mathrm{emf}}+\Delta \mu^{\mathrm{in}} / e}{\left(h / N e^{2}\right)+\mathcal{R}+\mathcal{R}_{i}},
$$

where the resistances $\mathcal{R}$ and $\mathcal{R}_{i}$ are given by Eqs. 31) and (30) respectively. Thus we see that, within an open geometry, the pump electromotive force adds to the voltage bias generated by the potential difference between the two electron reservoirs. This confirms the intuitive expectation that $\mathcal{V}_{\text {emf }}$ can be obtained from the the voltage difference $\Delta \mu^{\text {in }}$ needed to cancel the pump current [1, 6]. A striking difference between Eqs. (28) and (38) is the role of the contact resistance $h / N e^{2}$, which is absent in the case of a closed structure. Comparison of the underlying models suggests that the contact resistance disappears under the assumption that the flow of outgoing electrons on the left of the resistor-pump structure of Fig. 2 is identified with the the flow of incoming electrons from the right, and equivalently for electrons moving in the opposite direction. We conclude that, within a closed circuit of uniform width, there is no natural lower limit to the resistance that the electron current must face as it is generated by the electron pump. This result appears reasonable if one notes that contact resistances along the circuit are generated by narrow-wide contacts where the width of the wire (and thus the number of available electron channels) changes [13]. The denominators of Eqs. (28) and (38) suggest that the resistances which we have introduced should be additive. Unfortunately, the ratios $R / T$ and $\rho / \tau$ cannot be guaranteed to be additive in general. That is possible only in one dimension, where the ration $R / T$ is known to be additive for barriers compounded incoherently [14], or, for multichannel wires, in the particular case where the scattering probabilities are independent of the channel index $\left(R_{a b}=R / N^{2}, T_{a b}=T / N^{2}\right.$, and similarly for $\left.\rho_{a b}, \tau_{a b}\right)$. In such a case, the assumption of a common chemical potential for all incoming electrons $\left(\mu_{a}^{\text {in }}=\mu_{L, R}^{\text {in }}\right.$ for all $\left.a \in L, R\right)$ automatically guarantees an outgoing population with a common chemical for all outgoing electrons $\left(\mu_{b}^{\text {out }}=\mu_{L, R}^{\text {out }}\right.$ for all $\left.b \in L, R\right)$. Within that scheme, the assumption of a common chemical potential for all electrons moving in a given direction [see Eq. [15] is internally consistent in the sense that a scenario may be conceived where the outgoing population from a barrier or pump is guaranteed to be a suitable incoming population for the following obstacle. Interestingly, it is also in the channel-independent scattering limit where the resistance defined in Ref. [15] becomes additive and equivalent to the resistance defined in Eq. (31). On closer inspection, one realizes that the assumption of channel-independent scattering is hard to justify within an independent electron picture, where no naturally additive resistance can be defined for multichannel wires without invoking impurity averaging. In particular, such a hypothesis is not satisfied by the pipeline model invoked above, since its transmission depends on the perpendicular energy. We conclude that the question of the definition of an electron pump internal resistance is directly connected to the discussion on the additivity of resistances in multichannel wires. As long as this fundamental issue is not satisfactorily resolved, the transport equations (28) and (38) [as complemented by (29), (30), and (31)] which we have derived will have to be viewed as approximations obtained from a reasonable and appealing scheme. This research has been supported by the MCyT (Spain) under Grant No. BFM2001-0172, the EU RTN Programme under Contract No. HPRN-CT-200000144, and the Ramón Areces Foundation.

* Electronic address: miguel.rey@uam.es

$\dagger$ Electronic address: fernando.sols@uam.es

[1] M. Switkes, C. M. Marcus, K. Campman, and A. C. Gossard, Science 283, 1905 (1999).

[2] D. J. Thouless, Phys. Rev. B 27, 6083 (1983); Q. Niu, Phys. Rev. Lett. 64, 1812 (1990).

[3] C.A. Stafford and Ned S. Wingreen, Phys. Rev. Lett. 76, 1916 (1996).

[4] M.H. Pedersen and M. Büttiker, Phys. Rev. B 58, 12993 (1998).

[5] P. Brouwer, Phys. Rev. B 58, R10135 (1998).

[6] M. Wagner and F. Sols, Phys. Rev. Lett. 83, 4377 1999); F. Sols and M. Wagner, Ann. Phys. (Leipzig) 9, 776 (2000).

[7] S. W. Kim, Phys. Rev. B 66, 235304 (2002).

[8] J. Lehmann, S. Kohler, P. Hänggi, and A. Nitzan, J. Chem. Phys. 118, 3283 (2003).

[9] R. Landauer, IBM J. Res. Dev. 1, 223 (1957); Phil. Mag. 21, 863 (1970); M. Büttiker, Phys. Rev. Lett. 57, 1761 (1986).

[10] M. Büttiker, Phys. Rev. B 33, 3020 (1986).

[11] A. Stern, Y. Imry, Y. Aharonov, Phys. Rev. A 41, 3436 (1990).

[12] F. Sols and J. Sánchez-Cañizares, Superlatt. and Microstruct. 25, 627 (1999).

[13] Y. Imry, in Directions in Condensed Matter, G. Grinstein and E. Mazenko, eds. (World Scientific, Singapore, 1986).

[14] S. Datta, Electronic Transport in Mesoscopic Systems (North-Holland, Amsterdam, 1995).

[15] M. Büttiker, Y. Imry, R. Landauer, and S. Pinhas, Phys. Rev. B 31, 6207 (1985). 